\title{
Ito versus Stratonovich revisited
}

\author{
J. Smythe, F. Moss, \\ Department of Physics, University of Missouri-St. Louis, St. Louis, MO 63121, USA \\ P. V. E. McClintock, \\ Department of Physics, University of Lancaster, Lancaster LAl 4YB, UK \\ D. Clarkson, \\ Department of Mathematics, University of Missouri-St. Louis, St. Louis, MO 63121, \\ USA.
}

It is shown that a digital simulation of a noise induced phase transition using an algorithm consistent with the Ito stochastic calculus is in agreement with the predictions of that theory, whereas experiments with an analogue simulator yield measured results in agreement with the predictions of the Stratonovich theory. 
In this letter we report the results of an essentially experimental investigation of a model noise induced phase transition (NIPT) from the detailed points of view provided by two theoretical interpretations (due to Ito and Stratonovich) of the white noise process. Much has been written regarding these interpretations, and in the words of Van Kampen "... the discussion [sic] continues and threatens to grow to grotesque proportions". The interested reader will find a clear and succinct review in his papers [1]. Others [2, 3] have also contributed, but our citations here are by no means complete. A fundamental work is the paper by Mortensen [4]. Unfortunately "the discussion" has been carried on exclusively by theoreticians. At the risk of further inflating the already grotesque proportions, we wish to contribute the first (to our knowledge) experimental measurements able to distinguish between predictions based on the two interpretations. Our results are in substantial agreement with the views propounded by Van Kampen [1] and by West et al. [3], for the case of a dynamical system subject to external, multiplicative noise.

NIPTs were first studied by Horsthemke and Lefever [5-7] in certain model, nonlinear systems subject to environmental noise. They found that in such systems new, statistically favored, stationary states, which were unknown to the deterministic system, appear when the noise intensity exceeds some critical value. Here we shall exploit the fact that the Ito and Stratonovich interpretations lead to different predictions for the value of this critical noise intensity.

We are interested in the NIPT which appears in a genetic model [6] represented by

$$
d X / d t=\frac{1}{2}-X+\lambda_{t} X(1-X)
$$

where the external noise appears as the parameter $\lambda_{t} \rightarrow \lambda+\sigma \xi_{t}$. We take $\left\langle\lambda_{t}\right\rangle=\lambda$ and $\xi_{t} \xi_{t+\tau}=\delta(\tau)$ so that $\sigma \xi_{t}$ is a gaussian, white noise of variance $\sigma^{2}$, zero mean, and is the derivative of the Wiener process $W_{t}$. Eq. (1) thus becomes a stochastic differential equation

$$
d X=f(X, \lambda) d t+\sigma g(X) d W_{t}
$$

where $f(X, \lambda)=\frac{1}{2}-X+\lambda X(1-X)$, and $g(X)=X(1-X)$ for our model. Van Kampen has pointed out that eq. (2) is meaningless (he calls it a "pre-equation") until a prescription on how to integrate the second term on the right is adopted. If $G\left(W_{i}\right)$ is a function of the Wiener process, then the Ito convention is represented by a limit of the sum of finite differences, $\Sigma G\left(W_{i-1}\right)\left(W_{i}-W_{i-1}\right)$, where the function $G$ is evaluated at the left hand point $W_{i-1}$. The Stratonovich convention instead adopts a symmetric evaluation point, $\Sigma G\left[\left(W_{i}+W_{i-1}\right) / 2\right]\left[W_{i}-W_{i-1}\right]$. These sums have different limits and 
lead to different versions of the Fokker-Planck equation. The Ito version reads

$$
\partial_{t} \rho(X, t)=-\partial_{X} f(X) \rho(X, t)+\frac{1}{2} \sigma^{2} \partial_{X}^{2} g^{2}(X) \rho(X, t),
$$

and the Stratonovich is

$$
\partial_{t} \rho(X, t)=-\partial_{X}\left(f+\frac{1}{2} \sigma^{2} g^{\prime} g\right) \rho+\frac{1}{2} \sigma^{2} \partial_{X}^{2} g^{2} \rho
$$

where $\rho(X, t)$ is the time dependent probability density, and $g^{\prime}=\partial_{X} g(X)$. These have the wellknown stationary $\left(\partial_{t} \rho=0\right)$ solutions

$$
\rho_{s}=c g^{-\nu} \exp \left(\left(2 / \sigma^{2}\right) \int^{X}\left[f(z) / g^{2}(z)\right] d z\right)
$$

where $\nu=2(1)$ in the Ito (Stratonovich) versions, and $c$ is a normalization constant. For our model, eq. (5) results in

$$
\rho_{s}=[X(1-X)]^{-\nu} \times \exp \left\{-[2 X(1-X)]^{-1}-\lambda \ln [(1-X) / X]\right\} \times\left(2 / \sigma^{2}\right)
$$

which exhibits a single peak for $\sigma<\sigma_{c}$ and a double peak for $\sigma>\sigma_{c}$. For $\lambda=0$ the graph of $\rho_{s}$ is symmetric about $X=1 / 2$, and $\sigma_{c}^{(0)}=\sqrt{2}(2)$, in the Ito (Stratonovich) versions. For $|\lambda|>0$, one peak is enhanced and $\sigma_{c}>\sigma_{c}^{(0)}$. The phase diagram is the locus of $\sigma_{c}(\lambda)$.

We have digitally integrated eq. (1) using a finite difference approximation resembling the Ito prescription,

$$
X_{n+1}-X_{n}=\Delta X_{n}=\left(\frac{1}{2}-X_{n}\right) \Delta t+\beta_{t n} X_{n}\left(1-X_{n}\right),
$$

where a new $\beta_{t n}$ is chosen for each $n$ from a gaussian distribution with mean value $\lambda$ and variance $\sigma^{2} \Delta t$. A set of $N$ values $X_{t}$ are generated from each term in the time series $X_{t}=X_{0}+\sum_{n=1}^{N} \Delta X_{n}$ and the frequency that $X_{t}$ visited the interval between $X$ and $X+\Delta X$ was computed on the range $0 \leq X<1$ and stored. For $N$ sufficiently large, this average frequency distribution $\rightarrow \rho_{s}$. Typically, $N=2 \times 10^{5}, \Delta X=0.012$, and $\Delta t=0.05$. $X_{0}$ was invariably chosen as the steady state solution of eq. (1) for the deterministic $(\sigma=0)$ case,

$$
X_{0}=(1 / 2 \lambda)\left[\lambda-1+\left(1+\lambda^{2}\right)^{1 / 2}\right]
$$

to minimize initial transient effects. Some example densities from this simulation are shown in fig. 1(a) where for $\lambda=0$ it is clear that $\sigma_{c}^{(0)}$ is consistent with the Ito prediction. Fig. 1(b) shows examples for $|\lambda|>0$. By computing many such densities for various 
choices of $X$ we were able to generate the simulated data shown in the phase diagram, fig. 2, by the solid circles. The error bars were estimated from the difficulty of identifying that density with a range of $\simeq$ zero slope. The calculated result, shown by the solid curve marked "Ito", was obtained from eq. (6) (with $\nu=2$ ). The agreement is quite good considering the approximate nature of the simulation. No adjustable parameters were used either in generating the simulated data or the theoretical curve. We believe this demonstrates that the Ito formulation is correct from a mathematical point of view. It does not, however, tell us how nature actually behaves.

The authors of refs. [1,3] reach identical conclusions: Since the Stratonovich representation can be shown to result from the white noise limit of a real noise (i.e. band limited or nonzero time correlated) problem [8,9], then it should accurately describe the results of experiments on real dynamical systems. We have found this conclusion to be absolutely correct by actually measuring density functions on a real analogue simulator of eq. (I), driven by real noise. The noise correlation time was, however, much smaller than the dynamical response times of the system within the range over which the simulator was operated, so that a white noise description is expected to be a good approximation.

The simulator is shown in fig. 3. It is constructed entirely of analogue components except for the computer, which functions simply as an instrument for measuring and averaging the density of the noisy voltage $X(t)$. All sum and difference operations, as well as the two integrations are accomplished with quite standard operational amplifier circuits. The two multiplications were done with commercially available analogue multipliers*.

The simulator is accurate to $\simeq 3.8 \%$ in the sense that its steady state response with $\sigma=0$ is in agreement with eq. (7). The slow integrator has a time constant of $3 \mathrm{~s}$, and serves to stabilize the circuit on $\langle X(t)\rangle \simeq X_{0}$. We found that if the time constant was reduced much below about $1 \mathrm{~s}$, the accuracy was seriously impaired. The bandwidth of the slow integrator is therefore so narrow that the noise at its output is reduced by a factor $\simeq 2 \times 10^{-4}$. The noise $f(X, t)^{\dagger}$ was independently integrated by the fast integrator and subsequently added to $\langle X(t)\rangle$ at the output, so that $X(t)=\langle X(t)\rangle+\int f(X, t) d t$. The circuit was designed with a scale factor of unity, so that $X(t)$ in volts is numerically comparable to the results of eqs. (1) and (7).

The densities were measured by digitizing 4096 points in a time series of the voltage $X(t)$. The frequency that $X(t)$ visited the region between $X$ and $X+\Delta X$ was then

${ }^{*}$ Analog Devices, type AD534, Norwood MA 02062, USA.

${ }^{\dagger}$ Electrical engineers will recognize this closed loop circuit as a servo mechanism with error signal $f(X, t)$. Therefore when $\langle X(t)\rangle \equiv X_{0}$ then $\langle X(t)\rangle \equiv 0$, and this self regulation process operates on a time scale determined by the slow integrator time constant. 
computed for $0 \leq X<1$. The first time series was erased and a second obtained with the second frequency being added to the first. This process was repeated, typically 2048 times, whence the average frequency $\rightarrow \rho_{s}(X)$. Examples are shown in fig. 4 by the continuous curves compared to the calculated results (solid circles) from eq. (6). Fig. 4(a) shows a measurement for $\sigma \simeq \sigma_{c}=2$ compared to the Stratonovich result (flat curve) and the Ito result (double peaks) calculated for the same $\sigma$. Fig. 4(b) shows examples for $\sigma<\sigma_{c}$ and $\sigma>\sigma_{c}$ where the calculated results are always Stratonovich. For each value of $\lambda$, we were able to find $\sigma \simeq \sigma_{c}$ by searching for a density with a single region of approximately zero slope which separated the single peak set from the double peak set. The results are shown in fig. 2 by the open circles, and are in excellent agreement with the Stratonovich phase diagram.

We found that these results were independent of the correlation time of the noise, so long as this was kept smaller than about $3 \mathrm{~ms}$. (Most of the data were obtained with the noise generator band limited to an upper cutoff frequency of $300 \mathrm{~Hz}$, though we tested the results for cutoffs as low as $60 \mathrm{~Hz}$ and as high as $1500 \mathrm{~Hz}$.) This result indicates that the dynamical system sees the noise as approximately white. In addition, changing the time constant of the fast integrator only caused a change in the noise amplitude at the integrator output, with no effect on the measured quantities, e.g. $\sigma_{c}$. The single error bar shown in fig. 2 for these data, represents a systematic error related to the accuracy of the simulator. "Detuning" the simulator to reduce the accuracy to $\simeq \pm 6 \%$ compared to eq. (7) resulted in an upward or downward shift of the entire data set of magnitude comparable to the error bar shown. Thus the Stratonovich phase line is nicely bracketed by our data.

We conclude that the Ito prescription for integrating the stochastic differential equation is mathematically correct in the sense that an algorithm which imitates it produces results in approximate agreement with the Ito calculations. The Stratonovich results, however, accurately describe what actually happens in nature.

We are grateful to Werner Horsthemke of the University of Texas at Austin for several stimulating discussions. 


\section{$\underline{\text { References }}$}

l. N.G. van Kampen, J. Stat. Phys. 24 (1981) 175;25 (1981) 431.

2. S. Pick, Physica 103A (1980) 630.

3. B. West, A. Bulsara, K. Lindenberg, V. Seshadri and K. Shuler, Physica 97A (1979) 211, 234.

4. R. Mortensen, J. Stat. Phys. 1 (1969) 271.

5. W. Horsthemke and R. Lefever, Phys. Lett. 64A (1977) 19.

6. R. lefever, in: Stochastic, nonlinear systems in physics, chemistry and biology, eds. L. Arnold and R. Lefever (Springer, Berlin, 1981) p. 127; W. Horsthemke, in: Stochastic, nonlinear systems in physics, chemistry and biology, eds. L. Arnold and R. Lefever (Springer, Berlin, 1981) p. 116.

7. K. Kitahara, W. Horsthemke, R. Lefever and Y. Inaba, Prog. Theor. Phys. 64 (1980) 1233, and references therein.

8. F. Wong and M. Zakai, Ann. Math. Stat. 36 (1965) 1560.

9. G. Blankenship and C. Papanicolaou, SIAM J. Appl. Math. 34 (1978) 437. 


\section{Figures}

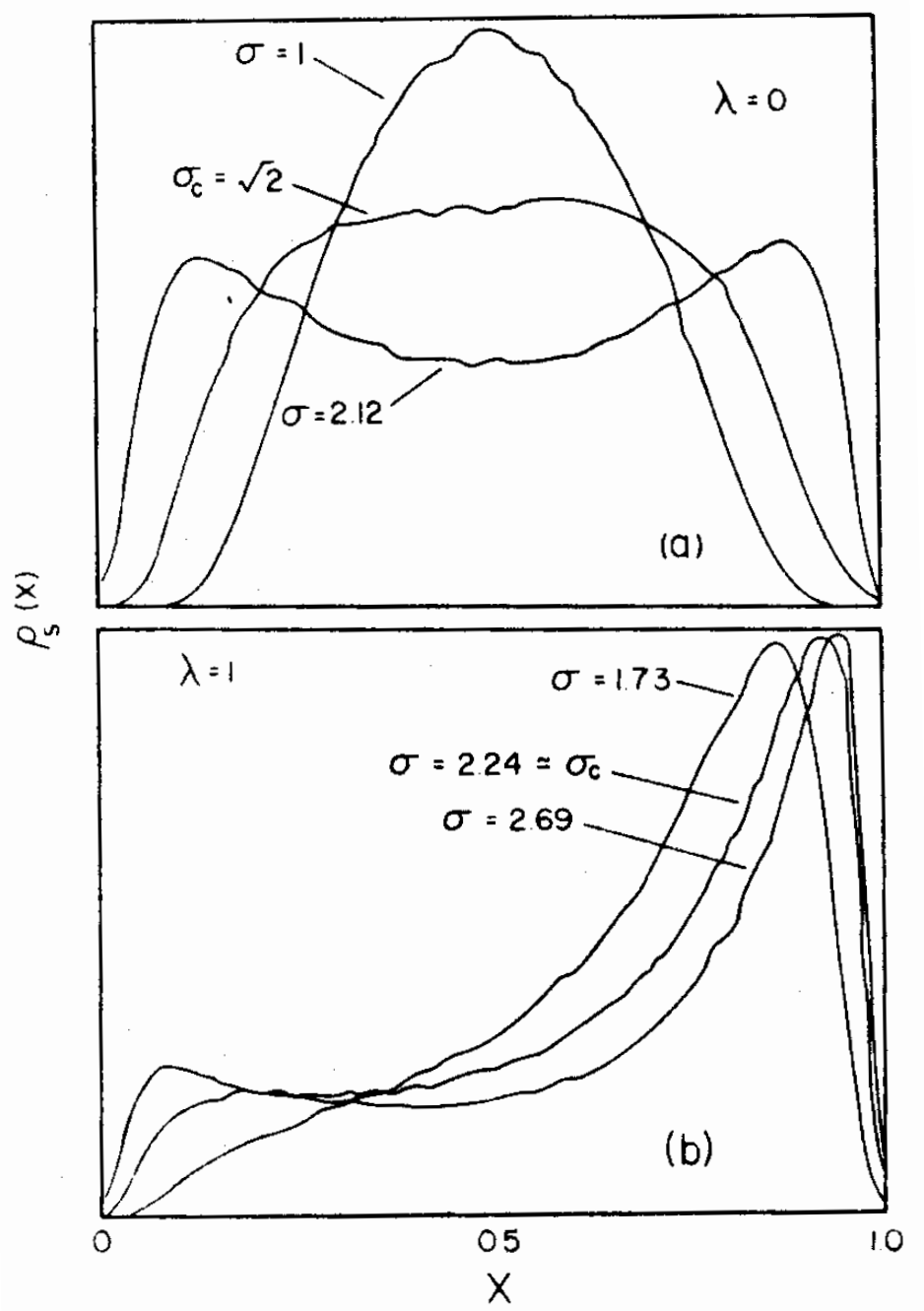

Figure 1: Digital simulation of Ito densities. (a) for $\lambda=0$ and (b) for $\lambda=1$ showing $\sigma<\sigma_{c}, \sigma \simeq \sigma_{c}$, and $\sigma>\sigma_{c}$. 


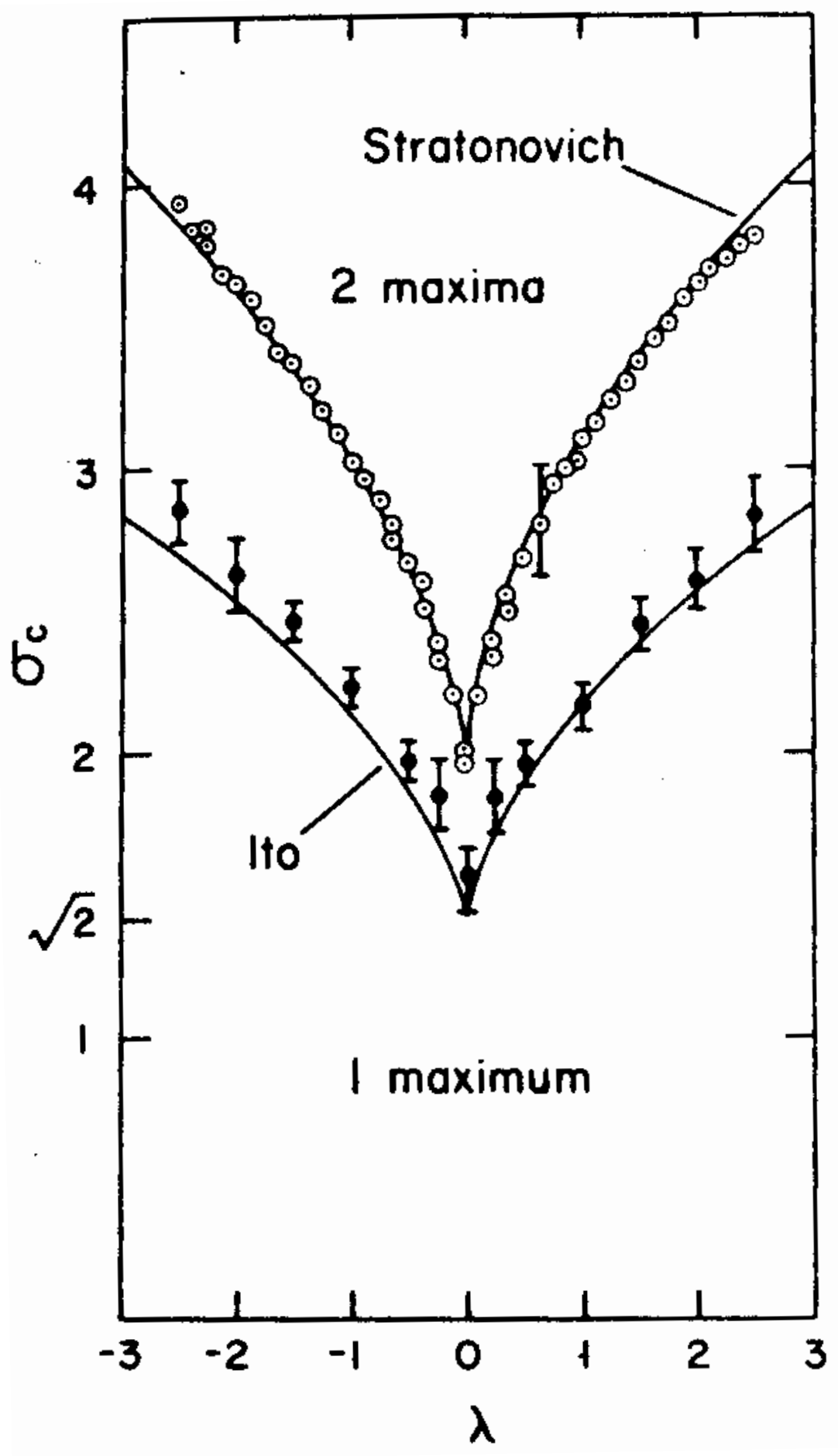

Figure 2: The phase diagram. The curves show results calculated from eq. (6). The solid circles show the results of the Ito digital simulation and the open circles are the experimental results obtained with the analogue simulator. 


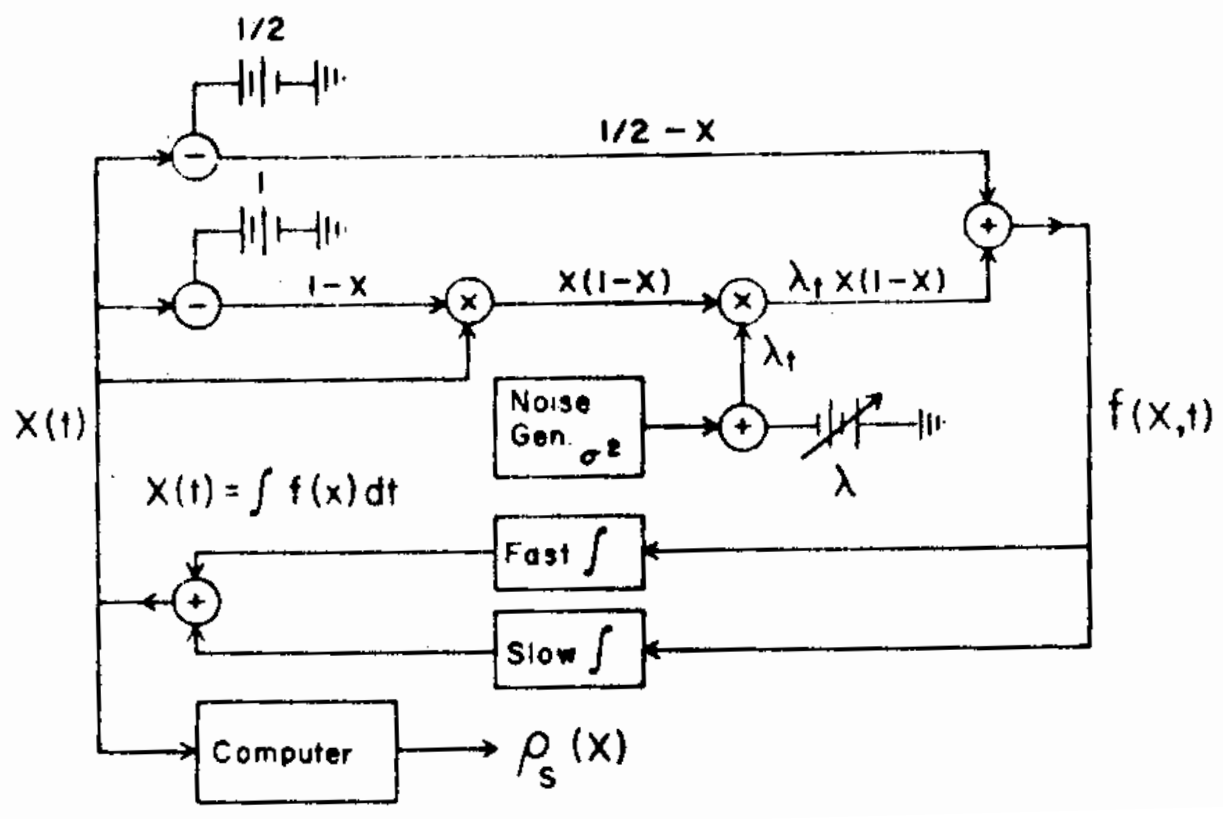

Figure 3: The analogue simulator. 


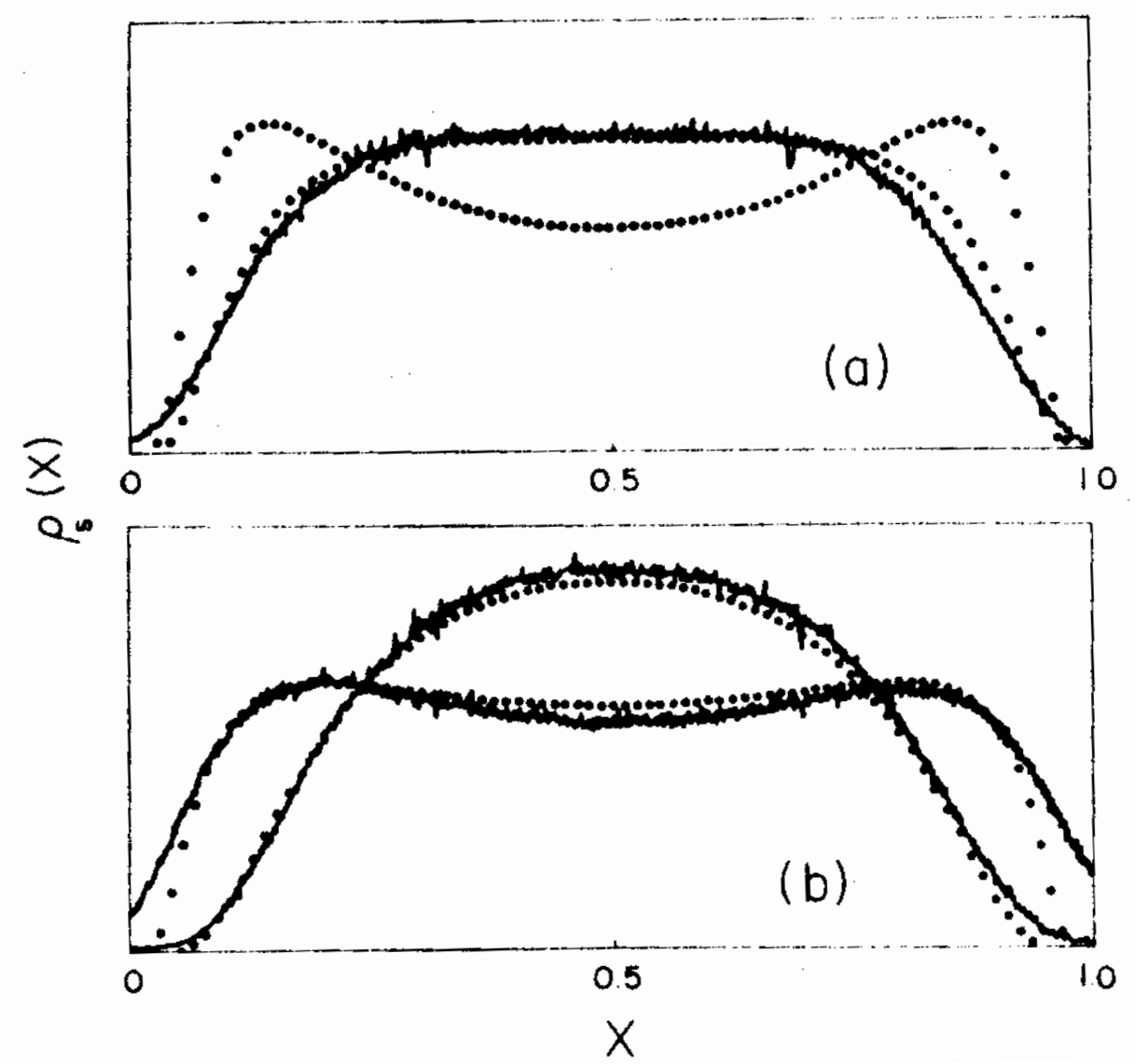

Figure 4: Densities measured at $\lambda=0$ on the analogue simulator (continuous curves) compared to those calculated from eq. (6) (solid circles). (a) For $\sigma=2.0$. The Stratonovich result for $\sigma_{c}^{(0)}=2$ is in good agreement with the measured density. The Ito prediction, also calculated for $\sigma=2$ is the double peaked curve. (b) Measured densities compared to the Stratonovich calculations for $\sigma=1.5$ (single peak) and for $\sigma=2.5$ (double peak). 